\title{
Energia livre da reação de adsorção de cádmio em latossolos ácricos ${ }^{1}$
}

\author{
Free energy of cadmium adsorption reaction in acric oxisols
}

\author{
Nivea Maria Piccolomini Dias ${ }^{2}$ Luís Reynaldo Ferracciú Alleoni ${ }^{3}$ \\ José Carlos Casagrande ${ }^{4}$ Otávio Antônio Camargo ${ }^{5}$
}

\section{RESUMO}

O cádmio é um metal pesado que pode ser adicionado ao solo por meio do lixo urbano ou industrial, lodo de esgoto e fertilizantes fosfatados. É facilmente absorvido e translocado pelas plantas, tendo potencial de entrar na cadeia alimentar humana. A persistência e a mobilidade do cádmio no solo é determinada pela intensidade da adsorção pelos colóides do solo. A avaliação da energia livre de adsorção de um elemento ao solo pode servir como medida da força da reação. Estudou-se a energia livre da reação de adsorção de cádmio em amostras superficiais (0$0,2 \mathrm{~m}$ ) e subsuperficiais (na maior expressão do horizonte B) de um Nitossolo Vermelho eutroférrico, um Latossolo Vermelho acriférrico e um Latossolo Amarelo ácrico, após a adição de 5, 10, 15, 25, 50, 75, 100, 125, 150, 175 e 200mg L $L^{-1}$ de cádmio. A adsorção de cádmio pelos solos foi espontânea, pois a energia livre apresentou valores negativos em todas as concentrações estudadas. Os valores de energia livre diminuíram com o aumento da dose de cádmio adicionada. $O$ Nitossolo apresentou maior energia livre do que os Latossolos, sobretudo na camada superficial. Os horizontes superficiais apresentaram maior energia livre para as reações de adsorção de cádmio dos que os subsuperficiais, provavelmente devido ao efeito da matéria orgânica, que apresenta alta afinidade pelo elemento.

Palavras-chave: material orgânica, óxido de ferro, ponto de efeito salino nulo.

\section{ABSTRACT}

Cadmium is a heavy metal that can be added to the soils through disposal of city wastes, sewage sludge and phosphatic fertilizers. Cd is easily absorbed and translocated by plants and has a great potential to enter the human food chain. The persistency and the mobility of Cd in soils is largely determined by the extent of its adsorption by soil particles. The evaluation of the free energy of Cd adsorption by the soil is a measurement of the reaction strength. Free energy of cadmium adsorption was studied in surface and subsurface samples from a Rhodic Kandiudalf, an Anionic "Rhodic" Acrudox and an Anionic "Xanthic" Acrudox, after addition of $5,10,15,25,50,75,100,125,150,175 \mathrm{e} 200 \mathrm{mg} \mathrm{L} L^{-1}$ of cadmium. Cadmium adsorption was considered spontaneous, since free energy was negative in all concentration. Free energy values decreased with the increase in concentration of added cadmium. The Alfisol showed higher free energy than the Oxisols, mainly in surface samples. For the same soil, surface horizons showed higher values of free energy than subsurface horizons, probably due to the effect of organic matter, whose affinity for cadmium is high.

Key words: organic matter, iron oxide, zero point of salt effect.

\section{INTRODUÇÃO}

O cádmio é um metal pesado, sendo assim considerado por apresentar número atômico 48 e massa específica $8,642 \mathrm{~g} \mathrm{~cm}^{-3}$. É utilizado para revestimento de materiais, em pigmentos de tintas e na indústria de plásticos, e pode ser adicionado ao solo por meio do lixo urbano ou industrial, lodo de esgoto e fertilizantes fosfatados. Por ser facilmente absorvido e translocado nas plantas, o $\mathrm{Cd}$ pode entrar na cadeia alimentar humana e causar sérios problemas de saúde, como anemia, hipertensão, enfisema pulmonar, disfunções gástricas e intestinais. A persistência e a mobilidade

${ }^{1}$ Parte da Dissertação de Mestrado da primeira autora, apresentada ao Programa de Pós-graduação em Solos e Nutrição de Plantas ESALQ/USP

${ }^{2}$ Doutorando em Ciências, Centro de Energia Nuclear na Agricultura/USP, CP 96, 13400-970, Piracicaba-SP.

${ }^{3}$ Professor Associado, Departamento de Solos e Nutrição de Plantas, ESALQ/USP, CP 9, 13418-900, Piracicaba-SP. E-mail: 1rfalleo@esalq.usp.br. Autor para correspondência.

${ }^{4}$ Professor Adjunto, Departamento de Recursos Naturais e Proteção Ambiental - UFSCar, CP 153, 13600-970, Araras-SP.

${ }^{5}$ Pesquisador, Centro de Solos e Recursos Agroambientais - IAC, CP 28, 13001-970, Campinas-SP. 
de cádmio no solo é determinada pela intensidade da adsorção pelos colóides, sendo o $\mathrm{Cd}$ considerado relativamente móvel no perfil dos solos, em comparação com outros metais pesados (PARDO, 2000, KIM \& KIM, 2001). Os atributos do solo que mais influenciam a capacidade de adsorção de cádmio no solo são os teores de matéria orgânica e de óxidos de $\mathrm{Fe}$ e $\mathrm{Al}$, a capacidade de troca de cátions, o pH, a força iônica da solução, a superfície específica e a composição mineralógica (KING, 1988).

O comportamento químico de metais pesados em solos altamente intemperizados é pouco estudado, embora haja vários artigos relacionados à retenção destes metais em sistemas puros, como caulinita e óxidos de Fe e de Al (NAIDU et al., 1998). Alguns pesquisadores estudaram as reações do cádmio em camadas superficiais de solos brasileiros, mas o conhecimento dos fatores que influenciam e/ou determinam estas reações ainda é restrito, principalmente em solos ácricos, que representam o extremo na escala de intemperização (ALLEONI \& CAMARGO, 1994). No horizonte B desses solos, o valor do ponto de carga zero (PCZ) em muitos casos supera o $\mathrm{pH}$ original, o que confere um balanço de carga positiva ao solo, que passa a atuar fundamentalmente como trocador de ânions. O fenômeno de reversão de carga ocorre quase sempre em profundidades maiores que $0,5 \mathrm{~m}$, pois, nesta condição, a matéria orgânica cujo PCZ é muito baixo (menor que 2), praticamente não atua. A maioria dos estudos com metais pesados, como o cádmio, restringem-se à camada arável dos solos $(0-0,2 \mathrm{~m})$. Entretanto, é importante conhecer também as reações dos metais nas camadas mais profundas, principalmente quando o horizonte B apresenta atributos eletroquímicos diferentes daqueles encontrados no horizonte A, como é o caso dos solos ácricos.

O estudo da adsorção de cádmio no solo possibilita o cálculo termodinâmico da energia livre das reações $\left(\Delta \mathrm{G}_{0}\right)$. As mudanças na energia livre que ocorrem quando um elemento é adsorvido podem servir como medida da força de determinada reação (SINGH, 1971). Seu sinal indica se a reação é espontânea ou não, em um sistema fechado, sob temperatura e pressão constantes. Seu valor também indica quão distante do equilíbrio está o estado inicial do sistema. Com a utilização de doses crescentes de cádmio, o valor da energia livre indica a magnitude da força de reação entre o metal e a superfície do adsorvente. Quanto maior seu valor, maior será a força da reação (ALLEONI \& CAMARGO, 1998). O objetivo deste trabalho foi estudar a energia livre da reação de adsorção de cádmio em três solos do Estado de São Paulo, sendo dois deles ácricos.

\section{MATERIAL E MÉTODOS}

Foram utilizadas amostras superficiais (0$0,2 \mathrm{~m}$ ) e subsuperficiais, coletadas da camada de maior expressão do horizonte B de um Nitossolo Vermelho eutroférrico (NVef), um Latossolo Vermelho acriférrico (LVwf) e um Latossolo Amarelo ácrico (LAw), localizados na região norte do Estado de São Paulo, que correspondem, respectivamente, à Terra Roxa Estruturada, ao Latossolo Roxo ácrico e ao Latossolo variação Una ácrico, na classificação vigente até 1999. O Nitossolo possui potencial eletroquímico diferente dos Latossolos ácricos, por apresentar carga elétrica negativa líquida ao longo de todo o perfil, e por isso foi utilizado como referência.

Após a coleta, os solos foram secos ao ar e, posteriormente, tamisados em peneira com abertura de malha de dois milímetros. A caracterização das amostras foi feita conforme metodologia descrita por CAMARGO et al. (1986). Foram determinados o teor de carbono orgânico, por oxidação da matéria orgânica do solo com solução de dicromato de potássio, e os teores de óxidos $\left(\mathrm{Al}_{2} \mathrm{O}_{3}, \mathrm{SiO}_{2}\right.$ e $\left.\mathrm{Fe}_{2} \mathrm{O}_{3}\right)$, após ataque com ácido sulfúrico $(9 \mathrm{M})$. A retenção de cátions $(\mathrm{RC})$ corresponde à relação entre a CTC efetiva $(\mathrm{Ca}+\mathrm{Mg}+$ $\mathrm{K}+\mathrm{Al}$ ) e a porcentagem de argila. $\mathrm{O}$ ponto de efeito salino nulo (PESN), que corresponde ao PCZ determinado por titulação potenciométrica, foi obtido em três concentrações de $\mathrm{KCl}$, sendo o PESN o valor de $\mathrm{pH}$ no ponto de intersecção das curvas de titulação (ALLEONI \& CAMARGO, 1994). As cargas negativas permanente $\left(\sigma_{\mathrm{o}}\right)$ e variável $\left(\sigma_{\mathrm{H}}\right)$ foram quantificadas com base na maior seletividade do $\mathrm{Cs}^{+}$nas cavidades siloxanas dos argilominerais 2:1 que nas superfícies ionizáveis (ANDERSON \& SPOSITO, 1991). Os resultados das análises são apresentados na tabela 1 .

Para a obtenção dos valores de $\mathrm{Cd}$ adsorvido, pesaram-se $2,00 \mathrm{~g}$ de terra fina seca em estufa e adicionaram-se $20 \mathrm{~mL}$ de solução $0,0025 \mathrm{~mol} \mathrm{~L}^{-1}$ de $\mathrm{Ca}\left(\mathrm{NO}_{3}\right)_{2}$ contendo $\mathrm{Cd}\left(\mathrm{NO}_{3}\right)_{2}$ nas concentrações de $5,10,15,25,50,75,100,125$, 150,175 e $200 \mathrm{mg} \mathrm{L}^{-1}$ de cádmio. Em seguida, as soluções foram centrifugadas, e as determinações foram realizadas em triplicata.

Com os resultados do experimento de adsorção, foram calculados os valores de energia livre das reações por meio da equação : $\Delta \mathrm{G}_{\mathrm{o}}=\mathrm{RT}(\ln \mathrm{Cd}$ equilíbrio - $\ln \mathrm{Cd}$ adicionado), sendo $\Delta \mathrm{G}_{\mathrm{o}}=$ energia livre $\left(\mathrm{J} \mathrm{mol}^{-1}\right), \mathrm{R}$ (constante universal dos gases) $=$ $8,30 \mathrm{~J} \mathrm{~mol}^{-1} \mathrm{~K}^{-1}, \mathrm{~T}=298 \mathrm{~K}$. 


\section{RESULTADOS E DISCUSSÃO}

Os valores de cargas negativas variáveis $\left(\sigma_{H}\right)$ e permanentes $\left(\sigma_{0}\right)$ foram maiores no NVef, tanto em superfície como em profundidade (Tabela 1). As cargas variáveis eram esperadas, pois em solos tropicais os principais materiais responsáveis por sua geração são a matéria orgânica, a caulinita e os óxidos e os hidróxidos de Fe e de Al, cujas cargas são variáveis com o $\mathrm{pH}$ e com a força iônica do meio. As quantidades de $\sigma_{\mathrm{H}}$ diminuíram em profundidade, o que pode ser atribuído ao menor teor de matéria orgânica, principal componente gerador de cargas negativas nos solos. A presença de carga elétrica negativa permanente, embora pequena, pode ser explicada pela presença de argilominerais do tipo 2:1 nos solos. A quantidade dessas cargas foi maior no NVef do que nos Latossolos ácricos, devido ao seu menor grau de intemperização. Nesse solo, foram identificados, por difratometria de raios $\mathrm{X}$, minerais como clorita/ vermiculita (interestratificada) e vermiculita na fração argila do horizonte A, além de ilita e muscovita na fração silte no horizonte B (resultados não apresentados). A presença de argilominerais do grupo 2:1 contribui para a maior adsorção de $\mathrm{Cd}$ aos solos, como já observado por TANIGUCHI et al. (2000).

Em todas as amostras, a $\Delta \mathrm{G}$ decresceu com o aumento da concentração de cádmionio adicionado (Figura 1), o que indica que, quanto mais concentrada a solução, menos fortemente o elemento foi adsorvido. PERCIVAL et al. (1999) obtiveram o mesmo resultado com solos australianos semelhantes aos utilizados neste estudo. Os autores explicaram que a energia de retenção do $\mathrm{Cd}$ diminui à medida que os sítios de adsorção são saturados. Além disso, com a adição do sal, ocorre queda do pH e diminuição na adsorção específica do elemento às superfícies adsorvedoras. $O$ decréscimo na energia livre em altas doses foi observado também por ALLEONI \& CAMARGO (1998) para a adsorção de boro e por SILVEIRA et al. (1999) para a adsorção de cobre. Todos os valores de $\Delta \mathrm{G}$ foram negativos, o que mostra que a quantidade de cádmio em equilíbrio na solução foi sempre inferior ao valor adicionado, ou seja, a reação de adsorção de cádmio foi termodinamicamente espontânea.

Quanto maior o valor da energia livre, maior é a força de reação do cádmio pela superfície adsorvente. Nos três solos, os maiores valores médios em módulo foram determinados na camada superficial (0-0,2 m), provavelmente devido ao maior teor de matéria orgânica, onde o principal sítio de adsorção de cádmio é a superfície dos grupos carboxílicos e fenólicos. Nesses grupos, o radical $\mathrm{OH}$ apresenta-se quase sempre dissociado nas condições normais de $\mathrm{pH}$ do solo, o que permite a retenção eletrostática de $\mathrm{Cd}$. A formação de complexos húmicos é pouco provável, pois as constantes de estabilidade destes complexos com o Cd é muito baixa (ELZINGA et al., 1999, SAUVÉ et al., 2000). PETRUZZELLI et al. (1985), HOODA \& ALLOWAY (1994), ECHEVERRIA et al. (1998) e GRAY et al. (1998) correlacionaram maiores valores de cádmio adsorvido com altos teores de carbono orgânico dos solos. LI et al. (2001) observaram decréscimo na adsorção de $\mathrm{Cd}$ em 14 solos americanos após a queima da matéria orgânica.

O NVef apresentou maiores valores de energia livre do que a dos Latossolos, tanto em superfície como em profundidade. Nas doses de

Tabela 1. Atributos químicos e mineralógicos dos solos estudados.

\begin{tabular}{|c|c|c|c|c|c|c|c|c|c|c|c|c|c|}
\hline \multirow{2}{*}{ Horiz. } & \multirow{2}{*}{ PESN } & \multirow{2}{*}{$\mathrm{pH} \mathrm{CaCl}{ }_{2}$} & \multirow{2}{*}{$\Delta \mathrm{pH}$} & \multirow{2}{*}{$\mathrm{Ki}$} & $\sigma_{\mathrm{o}}$ & $\sigma_{\mathrm{H}}$ & CTC & $\mathrm{RC}$ & $\mathrm{C}$ & Argila & $\mathrm{Ct}$ & $\mathrm{Fe}$ & $\mathrm{Al}$ \\
\hline & & & & & \multicolumn{5}{|c|}{--------- mmolc kg-1 --------- } & \multicolumn{4}{|c|}{------------ $\mathrm{g} \mathrm{kg}^{-1}$------------- } \\
\hline & \multicolumn{13}{|c|}{ Nitossolo Vermelho eutroférrico } \\
\hline A & 3,6 & 5,6 & $-0,9$ & 1,42 & 30,2 & 63,1 & 167 & 131 & 26 & 820 & 249 & 298 & 183 \\
\hline \multirow[t]{2}{*}{ B } & 3,6 & 5,6 & $-0,7$ & 1,62 & 30,9 & 35,4 & 81 & 77 & 7 & 700 & 263 & 273 & 237 \\
\hline & \multicolumn{13}{|c|}{ Latossolo Vermelho acriférrico } \\
\hline A & 3,6 & 4,3 & $-0,9$ & 0,77 & 8,2 & 39,9 & 93 & 105 & 20 & 600 & 172 & 321 & 248 \\
\hline \multirow[t]{2}{*}{ B } & 5,6 & 4,9 & $+0,3$ & 0,75 & 4,7 & 16,1 & 22 & 13 & 5 & 640 & 216 & 338 & 257 \\
\hline & \multicolumn{13}{|c|}{ Latossolo Amarelo ácrico } \\
\hline A & 3,4 & 4,3 & $-0,5$ & 0,78 & 8,4 & 32,3 & 43 & 38 & 16 & 350 & 248 & 59 & 113 \\
\hline $\mathrm{B}$ & 6,0 & 5,9 & $+0,6$ & 0,79 & 1,3 & 18,4 & 14 & 7 & 6 & 450 & 196 & 134 & 172 \\
\hline
\end{tabular}

PESN: ponto de efeito salino nulo: valor de $\mathrm{pH}$ correspondente à intersecção de curvas de titulação potenciométrica em soluções de $\mathrm{KCl}$; $\Delta \mathrm{pH}=\mathrm{pH} \mathrm{KCl} \mathrm{1M} \mathrm{-} \mathrm{pH} \mathrm{H} \mathrm{H}_{2} \mathrm{O} ; \sigma_{\mathrm{o}}=$ carga negativa permanente; $\sigma_{\mathrm{H}}=$ carga negativa variável; $\mathrm{CTC}=$ capacidade de troca de cátions; $\mathrm{RC}=$ retenção de cátions; $\mathrm{C}=$ carbono orgânico; $\mathrm{Ct}=$ caulinita; $\mathrm{Fe}=$ óxido de ferro; $\mathrm{Al}=$ óxido de alumínio.

Ciência Rural, v. 33, n. 5, set-out, 2003. 


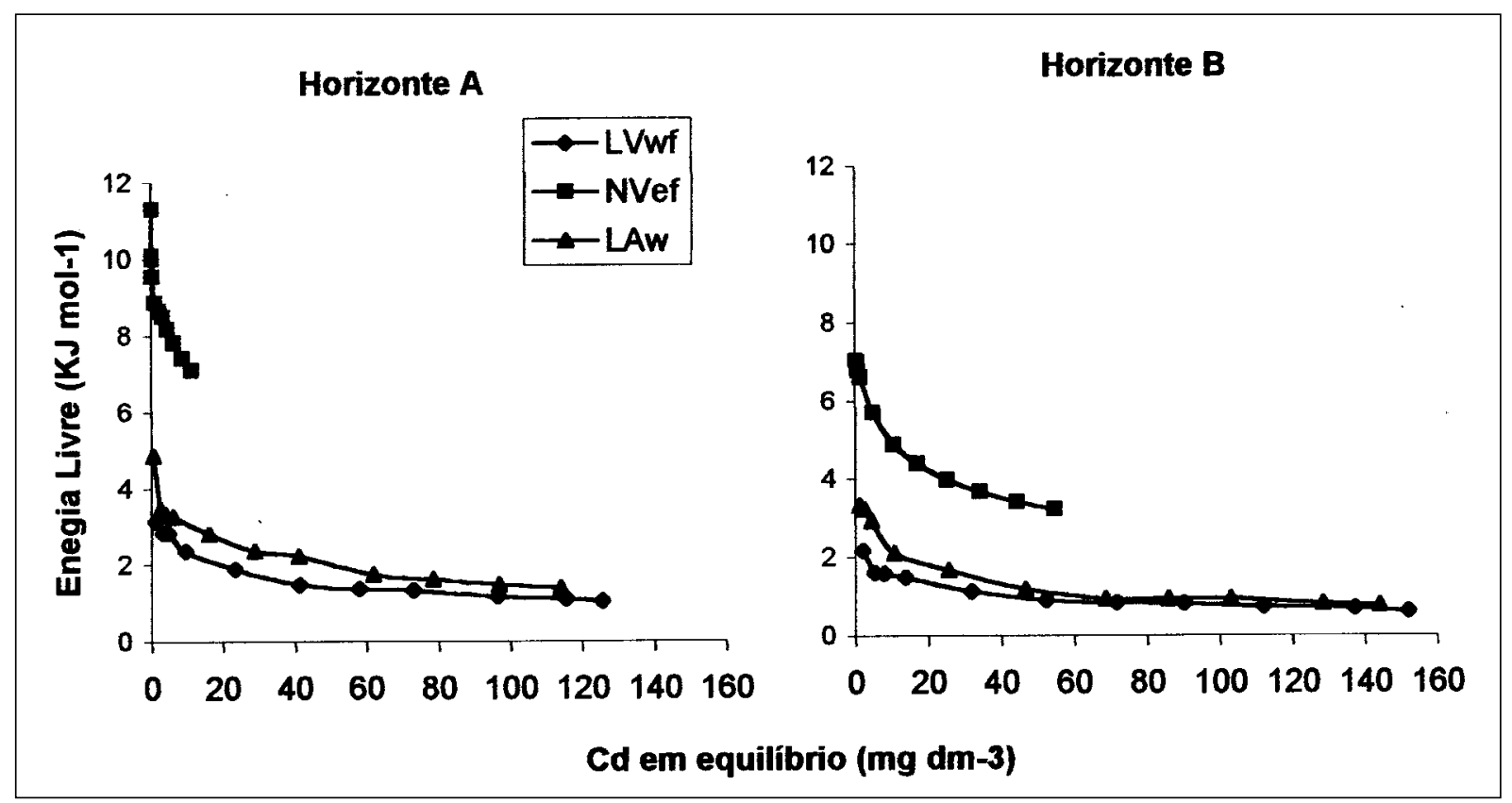

Figura 1 - Energia Livre (em módulo) das reações de adsorção de cádmio em camadas superficiais (a) e subsuperficiais (b) do Latossolo Vermelho acriférrico (LVwf), do Nitossolo Vermelho eutroférrico (NVef) e do Latossolo Amarelo ácrico (LAw).

cádmio adicionadas entre 5 e $200 \mathrm{mg} \mathrm{dm}^{-3}$, os valores de $\Delta \mathrm{G}_{\mathrm{o}}$ na camada superficial do Nitossolo variaram de $11 \mathrm{~kJ} \mathrm{~mol}^{-1}$, na menor concentração de cádmio adicionado, a $7 \mathrm{~kJ} \mathrm{~mol}^{-1}$, na maior dose. Em profundidade, os valores de energia livre variaram de 7 a $3 \mathrm{~kJ} \mathrm{~mol}^{-1}$ entre a menor e a maior dose de $\mathrm{Cd}$, respectivamente. Já os dois Latossolos apresentaram valores de energia livre semelhantes, com valores médios na camada superficial em torno de $4 \mathrm{~kJ} \mathrm{~mol}^{-1}$ na menor concentração do metal e próximos de $1 \mathrm{~kJ}$ $\mathrm{mol}^{-1}$ na maior dose. Em profundidade, os valores de energia livre foram inferiores, com valores médios de $2,5 \mathrm{~kJ} \mathrm{~mol}^{-1}$ e $0,7 \mathrm{~kJ} \mathrm{~mol}^{-1}$ na menor dose e na maior dose, respectivamente. Os maiores valores de energia livre determinados no NVef são conseqüência de sua constituição mineralógica, com predominância de caulinita e traços de argilominerais $2: 1$, resultado de seu menor estado de intemperização $(\mathrm{Ki}=1,74)$ (Tabela 1). O Nitossolo possui PESN inferior ao $\mathrm{pH}$ do solo, o que lhe confere carga líquida negativa em todo o perfil $(\Delta \mathrm{pH}=-0,9$ em superfície e - 0,7 em subsuperfície). KOOKANA \& NAIDU (1998) estudaram a adsorção de $\mathrm{Cd}$ num Alfissolo e num Oxissolo, enquanto PARDO (2000) comparou a adsorção de Cd em dois Alfissolos e um Andissolo. Em ambos os trabalhos, os autores associaram a maior quantidade de $\mathrm{Cd}$ adsorvido no Alfissolo à sua maior densidade de carga negativa. No Andissolo e no Oxissolo, a natureza da fração argila foi determinante na menor capacidade de retenção do elemento, sendo que no primeiro solo predominou a alofana, e no segundo óxidos de ferro e de alumínio.

Os menores valores de $\Delta \mathrm{G}_{\mathrm{o}}$ dos horizontes subsuperficiais dos Latossolos (Figura 1) podem ser devidos ao balanço de carga positivo no horizonte $\mathrm{B}$, como resultado do alto grau de intemperismo $(\mathrm{Ki}=0,75$ no LVwf e $\mathrm{Ki}=0,79$ no LAw) (Tabela 1 ) e maior participação dos óxidos de ferro e de alumínio na definição das cargas. Os valores de PESN desses óxidos são altos (com variação de 6,5 a 8,5), e quanto maiores suas quantidades, maior a possibilidade de o PESN dos solos ser maior que seus valores de $\mathrm{pH}$. Nesse caso, ocorre redução na energia de retenção do elemento. NAIDU et al. (1994) também determinaram os menores valores de cádmio adsorvido em solos altamente intemperizados e relataram que a menor adsorção é atribuída à alta superfície de carga positiva e à baixa densidade de carga negativa, ambas provenientes das mineralogias oxídicas e alofânicas dos solos.

A adsorção de metais pesados em óxidos e hidróxidos é governada por ligação covalente e específica (LOTHENBACH et al., 1997). Os óxidos de Fe são os que exercem a maior influência na energia de retenção de $\mathrm{Cd}$, seguidos pelos óxidos de $\mathrm{Al}$ e de Mn (DONG et al., 2000). Em baixas concentrações, o cádmio é adsorvido especificamente (TILLER et al., 1984). Os grupamentos hidroxila da superfície dos óxidos podem estar ligados a um, a dois ou até a três 
metais na estrutura do mineral, podendo formar complexos mono, bi e tridentados com o cádmio (FONTES, 1996). Já em altas concentrações, é esperada adsorção não específica, porque depois da saturação dos sítios de alta afinidade, a concentração residual do metal é elevada em relação aos outros cátions, e o metal é capaz de competir pelos sítios de baixa afinidade (TILLER et al., 1984). A adsorção de Cd em óxidos de Fe e de Al cristalino ocorre em dois passos: uma rápida adsorção na superfície de troca, seguida por uma lenta reação, sendo caracterizada como substituição do íon na matriz (penetração do íon dentro da fase sólida por um processo de recristalização). AINSWORTH et al. (1994) sugeriram que o Cd é incorporado nas estruturas dos óxidos via recristalização. É citada também a difusão do íon metálico para dentro da estrutura dos óxidos (BRUEMMER et al., 1988 e AINSWORTH et al., 1994), possivelmente em pequenos poros (FISCHER et al., 1996).

Em solos com pH menor que 6,5, como os deste estudo, a magnitude da adsorção é controlada pela capacidade de troca de cátions isolada de cada constituinte do solo (ZACHARA et al., 1992). O NVef apresentou maior quantidade de caulinita e maior CTC (Tabela 1) e mais de um tipo de mineral 2:1. Como conseqüência, a energia de retenção do $\mathrm{Cd}$ foi maior, como mostram os valores de $\Delta \mathrm{G}_{\mathrm{o}}$ (Figura 1). Num estudo em três solos com características contrastantes (Rhodic Kandiustaf, Calcic Haploxeralf e Typic Hapludand), PARDO (2000) observou que o Haploxeralf, com pH 6,4 e maior CTC, adsorveu mais $\mathrm{Cd}$ do que os outros dois solos, cujas CTCs eram menores. MATOS et al. (2001), estudando a relação entre a mobilidade de cádmio com as características químicas e mineralógicas de solos brasileiros, também verificaram a forte influência da capacidade de troca de cátions na retenção e mobilidade do cádmio nos solos. A matéria orgânica e a composição mineralógica determinam as propriedades de carga do solo, que se refletem no valor da CTC e da densidade superficial de carga, principalmente em solos com carga variável (NAIDU et al., 1998).

\section{CONCLUSÕES}

A energia livre de adsorção de cádmio foi maior nas menores concentrações de cádmio em equilíbrio e nas amostras com maiores teores de matéria orgânica. As reações de adsorção de $\mathrm{Cd}$ são espontâneas, mesmo em Latossolos com predominância de cargas positivas.

\section{AGRADECIMENTO}

À Fundação de Amparo à Pesquisa do Estado de São Paulo (FAPESP), pela concessão da bolsa de mestrado à primeira autora.

\section{REFERÊNCIAS BIBLIOGRÁFICAS}

AINSWORTH, C.C. et al. Cobalt, Cadmium, and Lead sorption to hydrous iron oxide: residence time effect. Soil Science Society of America Journal, Madison, v.58, p.1615-1623, 1994.

ALLEONI, L.R.F.; CAMARGO, O.A. Ponto de efeito salino nulo de latossolos ácricos. Revista Brasileira de Ciência do Solo, Campinas, v.18, p.175-180, 1994.

ALLEONI, L.R.F.; CAMARGO, O.A. Energia livre das reações de adsorção de boro em solos. Revista de Agricultura, Piracicaba, v.73, p.319-330, 1998.

ANDERSON, S.J.; SPOSITO, G. Cesium-adsorption method for measuring acessible structural surface charge. Soil Science Society of America Journal, Madison, v.55, p.1569-1576, 1991.

BRUEMMER, G.W.; GERTH, J.; TILLER, K.G. Reaction kinetics of the adsorption and desorption of nickel, zinc and cadmium by goethite. I. Adsorption and diffusion of metals. Journal of Soil Science, Oxford, v.39, p.37-52, 1988.

CAMARGO, O.A. et al. Métodos de análise química, mineralógica e física de solos do Instituto Agronômico de Campinas. Campinas : IAC, 1986. 94p.

DONG, D. et al. Adsorption of $\mathrm{Pb}$ and $\mathrm{Cd}$ onto metal oxides and organic material in natural surface coatings as determined by selective extractions: new evidence for the importance of $\mathrm{Mn}$ and Fe oxides. Water Research, Oxford, v.34, p.427436, 2000 .

ECHEVERRIA, J.C.; MORERA, M.T.; MAZKIARAN, C. Competitive sorption of heavy metal by soils. Isotherms and fractional factorial experiments. Environmental Pollution, Oxford, v.101, p.275-284, 1998.

ELZINGA E.J.; GRINSVEN, J.J.M. van; SWARTJES, F.A. General purpose Freundlich isotherms for cadmium, copper and zinc in soils. European Journal of Soil Science, Oxford, v.50, p.139149, 1999.

FISCHER, L. et al. Atomic force microscopy (AFM) investigations of the surface topography of a multidomain porous goethite. European Journal of Soil Science, Oxford, v.47, p.329-334, 1996.

FONTES, M.P.F. Caracterização da carga elétrica superficial de partículas do solo. In: CONGRESSO LATINO AMERICANO DE CIÊNCIA DO SOLO, 13., 1996, Águas de Lindóia, Brasil. Trabalhos... Piracicaba : SBCS/SLCS,1996. CD-Rom.

GRAY, C.W. et al. Sorption de cadmium from some New Zealand soils: effect of $\mathrm{pH}$ and contact time. Australian Journal of Soil Research, Melbourne, v.36, p.199-216, 1998.

HOODA, P.S.; ALLOWAY, B.J. Effects of time and temperature on the bioavailability of $\mathrm{Cd}$ and $\mathrm{Pb}$ from sludge-amended soils. Journal of Soil Science, Oxford, v.44, p.97-110, 1994. 
KIM, S.O.; KIM, K.W. Monitoring of electrokinetic removal of heavy metals in tailing-soils using sequential extraction analysis. Journal of Hazardous Materials, Amsterdan, v.85, p.195-211, 2001.

KING, L.D. Retention of metals by several soils of the Southeastern United States. Journal of Environmental Quality, Madison, v.17, p.239-246, 1988

KOOKANA, R.S.; NAIDU, R. Effect of soil solution composition on cadmium transport through variable charge soils. Geoderma, Amsterdan, v.84, p.235-248, 1998.

LI, Z.B. et al. Adsorption of cadmium on biosolids-amended soils. Journal of Environmental Quality, Madison, v.30, p.903-911, 2001.

LOTHENBACH, B.; FURRER, G.; SCHULIN, R. Immobilization of heavy metals by polynuclear aluminium and montmorillonite compounds. Environmental Science Technology, Washington, v.31, p.1452-1462, 1997.

MATOS, A. T. et al. Mobility of heavy metals as related to soil chemical and mineralogical characteristics of Brazilian soils. Environmental Pollution, Oxford, v.111, p.429-435, 2001.

NAIDU, R. et al. Ionic-strength and $\mathrm{pH}$ effects on the sorption of cadmium and the surface charge of soils. European Journal of Soil Science, Oxford, v.45, p.419-429, 1994.

NAIDU, R.; SUMNER, M.E.; HARTER, R.D. Sorption of heavy metals in strongly weathered soils: an overview. Environmental Geochemistry end Health, Dordrecht, v.20, p.5-9, 1998.

PARDO, M.T. Sorption of lead, copper, zinc, and cadmium by soils: Effect of nitriloacetic acid on metal retention. Communications in Soil Science and Plant Analysis, New York, v.31, p.31-40, 2000.
PERCIVAL, H.J.; SPEIR, T.W.; PARSHOTAM, A. Soil solution chemistry of contrasting soils amended with heavy metals. Australian Journal of Soil Research, Melbourne, v.37, p.9931004, 1999.

PETRUZZELLI, G.; GUIDI, G.; LUBRANO, L. Ionic strength effect on heavy metal adsorption by soil. Communications in Soil Science and Plant Analysis, New York, v.16, p.971-986, 1985.

SAUVÉ, S. et al. Speciation and complexation of cadmium in extracted soil solutions. Environmental Science \& Technology, Washington, v.34, p.291-296, 2000.

SILVEIRA, M.L.A. et al. Energia livre da reação de adsorção de cobre em latossolos ácricos. Scientia Agricola, Piracicaba, v.56, p.1117-1122, 1999. (Suplemento)

SINGH, M. Retention of added copper by two soils as affected by organic matter, $\mathrm{CaCO}_{3}$ and exchangeable ions. Geoderma, Amsterdan, v.5, p.219-227, 1971.

TANIGUCHI, S.; YAMAGATA, N.; SAKURAI, K. Cadmium adsorption on hydroxy-aluminosilicatemontmorillonite complex as influenced by oxalate and citrate. Soil Science and Plant Nutrition, Tokyo, v.46, p.315-324, 2000 .

TILLER, K.G.; GERTH, J.; BRUMMER, G. The relative affinities of $\mathrm{Cd}$, $\mathrm{Ni}$ and $\mathrm{Zn}$, for different soil clay fractions and goethite. Geoderma, Amsterdan, v.34, p.17-35, 1984.

ZACHARA, J.M. et al. Cadmium sorption to soil separates containing layer silicates and iron and aluminum oxides. Soil Science Society of America Journal, Madison, v.56, p.19741084, 1992. 